\title{
Amplatzer Amulet Left Atrial Appendage Occluder: A Step-By-Step Guide to Device Implantation
}

\author{
Christopher Ellis ${ }^{1}$ \\ ${ }^{1}$ Vanderbilt University Medical Center
}

January 24, 2022

\begin{abstract}
Recently receiving FDA approval, the Amplatzer Amulet LAA occluder device (Abbott Medical Inc) provides a dual seal mechanism alternative to the commercially available Watchman FLX or Watchman 2.5 device for embolic stroke prevention in patients with non-valvular atrial fibrillation (NVAF). This Step-by-Step review will cover patient selection, pre-procedure imaging assessment, device sizing, device preparation, implant target position assessment, implant technique and troubleshooting, and immediate post implant follow-up.
\end{abstract}

\section{Introduction}

Atrial fibrillation (AF) confers an increased risk of embolic events stratified by $\mathrm{CHA}_{2} \mathrm{DS}_{2}$-VASc score which informs consideration of risk modifying therapy with systemic oral anti-coagulation (OAC). Many patients with increasing $\mathrm{CHA}_{2} \mathrm{DS}_{2}$-VASc score are unsuitable for long term OAC due to real or perceived bleeding risk. The first percutaneously inserted intra-cardiac left atrial appendage occlusion (LAAO) device brought to market was the PLAATO device in 2001, subsequently withdrawn in $2006^{1,2}$

The Watchman LAAO device was shown to be non-inferior to Vitamin K antagonists (VKAs) in the PROTECT-AF and PREVAIL trials and subsequently FDA approved in 2015 as an alternative for oral anticoagulation. The Watchman device (Boston Scientific Inc) is currently in its second-generation iteration as the Watchman FLX which was shown to be safer to implant and provides higher complete seal rates with lower DRT (device related thrombus) than its predecessor ${ }^{3-5}$.

The results of the Amplatzer Amulet device IDE (Abbott Medical, Inc.) trial against the first-generation Watchman 2.5 device was published in Circulation in 2020. The Amulet device is a self-expanding nitinol mesh lobe inserted into the LAA using 6-10 stabilizing wires and is connected to a polyester covered disk that seals the LAA ostium. It is available in 8 different sizes with a minimum LAA implant depth of $12 \mathrm{~mm}$. In comparison, the Watchman or Watchman FLX is a self-expanding nitinol frame semi covered by a polyethylene terephthalate (PET) membrane fabric held in by 10-12 anchors. The Amulet IDE trial randomized 1878 patients in an intention to treat analysis to either the Watchman 2.5 or the Amulet device. The Amulet device was shown to be as effective as the Watchman in the composite outcome of stroke, systemic embolism, or cardiovascular/unexplained death $(5.6 \%$ vs $7.7 \%$, difference $=-2.12,95 \%$ CI, -4.45 $0.21 ; \mathrm{p}<0.001$ for noninferiority). The procedural complications were higher in the Amulet group (4.5\% vs $2.5 \%$ ), and more frequent with early case numbers consistent with an operator learning curve. The overall safety of the 2 devices were otherwise similar.

The Amulet IDE trial defined technical device success as a peri-device jet of $<5 \mathrm{~mm}$ which occurred in $96 \%$ of Amulet cases and $94.5 \%$ of Watchman cases, with a complete occlusion rate of $63 \%$ for the Amulet occluder and $46.1 \%$ for the Watchman 2.5. The PINNACLE-FLX trial of the Watchman FLX similarly showed a peri-device leak of $<5 \mathrm{~mm}$ in $100 \%$ of patients, with $7.4 \%$ having a residual leak between 0 and 5 
$\mathrm{mm}^{10}$. Recent data suggest residual leak of even $3 \mathrm{~mm}$ or beyond confers an increased risk of embolic events, and more study is needed to determine if the Amulet does provide a better seal ${ }^{6,7}$.

The increased rate of complete occlusion at the time of the procedure allowed more patients who received the Amulet occluder to be discharged off anticoagulation however at 12 months follow up most (>90\%) patients from both groups were off anticoagulation. Pericardial effusion rates in the first 30 days post LAAO with Amulet were balanced by lower DRT rates despite a DAPT only post implant regimen in the majority of subjects. Direct comparisons of Watchman FLX to Amulet as regards complete LAA closure by CT angiography versus TEE support a comparable occlusion rate ${ }^{8,9}$.

Achieving a complete seal with Amulet is dependent both on the co-axial deployment of a stable and modestly compressed 'lobe' inserted into the LAA neck followed by placement of the sealing disc with circumferential contact to the left atrial wall ideally from the tip of the left pulmonary vein-LAA ridge to the edge of the mitral valve annulus (see Figure 1). The lobe should be anchored with $2 / 3$ of the lobe height below the axis of the left circumflex coronary artery. When engaged properly the disc will then sit with a slight angulation from the lobe with separation and elliptical or concavity visible on X-Ray indicating proper cupping of the device to provide complete occlusion. Features of device selection, preparation, implant and release will be detailed below.

\section{Pre-procedure LAA Anatomy Assessment and Patient Selection}

Patient selection for LAAC with Amulet remains in line with CMS coverage and FDA approval for patients with NVAF and a $\mathrm{CHA}_{2} \mathrm{DS}_{2}$-VASc 3-9 and a compelling indication to avoid long term systemic OAC. LAA anatomy assessed pre-operatively with either gated cardiac CTA or TEE can guide device selection, sizing, and is used to exclude LAA thrombus prior to device placement. In cases with limited coaxial implant depth $(<15 \mathrm{~mm})$, Amulet may be a preferred option as the uncompressed lobe height is $7.5 \mathrm{~mm}$ with $16-22 \mathrm{~mm}$ size devices, and $1 \mathrm{~cm}$ with $25-34 \mathrm{~mm}$ devices).

The Amulet device overall sits more proximally than Watchman, and as such can provide adequate LAA closure in situations where large mid-cavitary pectinates may push an LAAC device forward. Cases with an extremely elliptical LAA orifice with extensive pits along the LPV-LAA ridge which may remain uncovered by Watchman FLX, also may be appropriate to select Amulet up front. Regarding patient medical history, patients with an obligation to dual anti-platelet therapy (DAPT) for coronary artery disease, may be ideal for Amulet as this is the FDA labeled post implant drug regimen. Patients with intracranial hemorrhage history may also favor Amulet to avoid post implant OAC/NOAC. Frailty and renal dysfunction may favor Watchman FLX given implant may require less reliance on contrast load or X-Ray, can be done with intracardiac echocardiography (ICE) only, and generally is associated with reduced total anesthesia or procedure time. As operators gain more experience with Amulet in the US, these factors may equilibrate, registry data will be needed. See Figure 2.

\section{Amulet Device Sizing and Selection}

The Amulet LAAC device comes in 8 different sizes $(16,18,20,22,25,28,31,34 \mathrm{~mm})$ and as such, a more tight range of landing zone measurement should be adhered to in order to avoid significant 'low' compression (embolization risk), or 'over' compression leading to potential for erosion or reduced anchor engagement. Additionally, this means the precision and accuracy of measurement of the landing zone for device sizing is more essential. The ostial measurement spans the tip of the LPV-LAA ridge to the LAA edge at the mitral annular aspect, from this line a second measurement is made $10-12 \mathrm{~mm}$ into the LAA, coaxial to the neck, for landing zone (LZ) measurement. The LZ is the primary factor in device size selection, provided the disc will be sufficient to cover the true LAA ostium. For $16-22 \mathrm{~mm}$ devices, the disc is $6 \mathrm{~mm}$ larger than the lobe, for $25-34 \mathrm{~mm}$ devices the disc is $7 \mathrm{~mm}$ larger than the lobe. The device is anchored by a series of paired wires either 6,8 or $10(12,16$, or 20 individual wires) in keeping with larger lobe sizes. Typical pre-procedure TEE Amulet measurements are shown in Figure 3.

The LZ may be relatively round, in which case sizing can be matched to the LZ range, or may be sized up 
one device. When the LZ is quite elliptical ( $>2: 1$ long to short axis), the largest width should be considered as the Amulet device size, and excessive oversizing may lead to an over compressed device (see Figure 4). There will be a maximum reasonable separation of the lobe and disc that can be achieved with a stable position, and the degree of 'overhang' of the LPV-LAA ridge may affect the expected position where Amulet may best sit. Cases leaving an overhang of the ridge on the disc with an angle $<90$ degrees may promote device related thrombi and attempts to re-orient the device should be taken (see Figure 5).

\section{Device Preparation}

The Amulet device delivery system is assembled prior to insertion into the Torque Vue 45-degree Amulet delivery sheath. Once the size of device is selected, either a $12 \mathrm{Fr}$ or $14 \mathrm{Fr}$ delivery sheath is prepped. Devices of $16-22 \mathrm{~mm}$ are best delivered through the $12 \mathrm{Fr}$ sheath, whereas the $28-34$ devices must be placed in a $14 \mathrm{Fr}$ sheath. A $25 \mathrm{~mm}$ Amulet can be implanted using either sheath though in our experience the 14Fr is preferrable (requires sheath adaptor). The delivery cable should be checked with clockwise torque to ensure the attachment screw on the Amulet device in the loading tube is tight prior to implant. A Tuohy and rotating 3 -way stopcock are attached to the insertion tool and side port. The device is then de-aired with a series of $60 \mathrm{cc}$ saline flushes which requires careful inspection, manual massage even tapping or flicking the tube. Pulsated injection of saline forward and backward through the tool while the device tip is advanced just to the edge of the loading tube and submerged until clear. Device preparation is typically done after transeptal and the Torque Vue sheath is in place in the LAA neck to minimize the time from device preparation to device insertion. See Video 1 on device preparation.

\section{Step By Step Amulet Implant Technique}

Patients referred for Amulet are typically at increased risk for AF related stroke, and as such, NOAC or OAC is continued until the $24 \mathrm{hrs}$ prior to device implant, with plans to transition to DAPT (dual anti-platelet therapy). Cases are currently performed largely with general anesthesia and TEE guidance provided by the cardiac anesthesia team. Vascular access is obtained from the right femoral vein approach, ideally with ultrasound guidance to avoid inadvertent arterial injury. Typical mid to inferior transeptal access location is adequate in most cases for Amulet placement as the target location for lobe placement is more proximal than the Watchman. The Torque Vue sheath is exchanged over a stiff wire either in the LA or the LSPV. TEE biplane 45-135 degree imaging is helpful to watch the sheath tip and orient to the LAA over a $6 \mathrm{Fr}$ pigtail catheter. A bare sheath should not be advanced without a pigtail or the Amulet in 'ball' position. When ready to deliver, a fluid-to-fluid connection is essential to the open end of the 12 or 14Fr Torque Vue sheath while bleeding back through the Touhy. The cable is advanced to push the device to the tip of the delivery sheath, after which the sheath is backed out until the Amulet forms the 'ball' (see Figure 6).

Biplane TEE is best for advancing the ball to the lobe landing zone position, and verifying that the ball is co-axial to the neck of the LAA. (see Video 2). The 'ball' position can be clearly seen on TEE as well and gradual advancing and counterclockwise sheath rotation is used until the ball is seen advancing past the LCX landmark. The 135-degree TEE is useful to then orient the ball in mid cavity aligned to the LZ. Cable is advanced slowly to the 'triangle' or 'lunar lander' shape after which, additional torquing on the sheath is discouraged. This is to avoid shearing or scraping of the LAA wall with the anchoring wires fully engaged. Forward pressure can be provided to avoid slipping of the lobe too proximal as it will slightly jump back towards the sheath when fully deployed, if lobe position is not ideal, lobe can easily be retracted to 'ball' shape again to re-orient (see Video $3 \mathrm{a}$ and $3 \mathrm{~b}$ ).

\section{Procedural Scenarios Warranting Repositioning}

When properly positioned, the Amulet will fulfil five key criterion for release, the "CLOSE" signs. Compression of the lobe, and lobe position $2 / 3^{\text {rd }}$ 's below the Left circumflex. Orientation of the device coaxial to the neck of the LAA, Separation of the disc and lobe with stability during tension testing, and Elliptical disc shape showing concavity. Performing an angiogram through the sheath (10-20mL contrast) with only the lobe deployed can be quite helpful to ensure proper lobe orientation, and no leaks $>3 \mathrm{~mm}$ before deploying the disc (see Figure 7). TEE views of the lobe deployed are essential to ensure the lobe is not slipping or 
shifting during deployment (see Video 4a and 4b).

The lobe should be carefully watched by the TEE operator while the disc is placed. Disc deployment is un-sheathing the first $2 / 3$ of the disc, then letting the tension off the Tuohy, allow the disc to be pulled in gradually to position with gentle forward pressure on the delivery cable. At this point, excess pressure should be avoided to prevent destabilizing the anchoring wires, particularly when the lobe has low to medium compression (see Video 5). Once the disc is deployed, the cable can be pulled back creating the 'football' shape of the disc and lock the valve on the delivery cable in place and observe for 30-60sec. This tension testing is mainly to ensure the lobe position does not change (see Video 6). In cases where the disc is too distal, or has a $<90$ degree angle abutting the LPV-LAA ridge as in figure 4, this same pull-back on cable to re-orient the disc in an attempt to cap the tip of the ridge is attempted, or full lobe recapture is needed (see Video 7).

After survey for any leaks and suitable position for release, the "CLOSE" signs are again reviewed, the cable then can be released with slight pull back tension and counterclockwise rotation, 8-10 turns typically. The disc should pull towards the lobe and cup into a concave shape indicating good apposition to the LA wall (see Video 8). Ideal device position by TEE is demonstrated in Video 9.

The following scenarios would warrant repositioning of the Amulet device for which the operator should never hesitate to perform:

Lobe too compressed and too deep (full engagement of distal LAA architecture).

Lobe position too proximal or unstable after tension test or disc placement.

Lobe not compressed.

Large leak (>3mm) of flow by Doppler US or contrast angiography around lobe.

Disc compression of pulmonary vein (L) or impinging on the mitral valve annulus (see Video 10).

Disc flat and in contact with lobe.

\section{Acute Complications and Management}

The major implant risk for Amulet is reported at $4.5 \%$ in the Amulet IDE, largely accounted for by early operator experience. The most likely acute emergency encountered during implant would be pericardial effusion requiring drainage. Having on site cardiothoracic surgery is advised in Amulet implant centers, also for the rare event of a device embolization into the left ventricle or ascending aorta. Snare retrieval of an Amulet is possible with a combination of large bore sheaths in the LA and a goose neck snare. Being able to manage an acute effusion with reversal of anti-coagulation and drain placement is a needed skill for all Amulet implanters, though often the effusion can present late (7-14 days post implant). Groin access complications can be minimized with ultrasound guidance during vascular access. Airway or TEE related complications are fortunately rare. Careful attention to transeptal access location, adherence to the "CLOSE" signs, cautious sheath and device movement in the LA-LAA, and proper device positioning are the best methods to avoid complications.

\section{Post Implant Imaging and Follow-Up}

After successful device release, we typically reverse the $120 \mathrm{un} / \mathrm{kg}$ heparin bolus with Protamine $50 \mathrm{mg}$ following a test dose. A figure of 8 suture is placed and sheath removed with manual hemostasis. A 4 hour down time is needed, after which we obtain a PA/Lateral CXR after ambulation. Though in clinical trials using the Amulet, a post-operative echocardiogram is protocol driven, we have found after $>100$ implants, that this rarely necessary unless clinical concerns arise regarding pain, delayed effusion or embolization. Most patients remain in observation overnight, with more recent cases aiming for same-day discharge. We arrange a follow up TEE at 90 days post implant in all patients and discharge on dual anti-platelet regimen with ASA $81 \mathrm{mg}$ and clopidogrel $75 \mathrm{mg}$ QD. NOAC or OAC is discontinued immediately post implant. Given 
concerns for late DRT, we continue to also image the device at 1 year post implant while the patient is on ASA or single anti-platelet therapy alone.

\section{Conclusions}

The Amulet LAA occlusion device is designed as a two-phase occluder with a distal anchoring lobe placed inside the neck of the LAA and a larger outer disc, designed to align to the anatomy of the main left atrial wall body outside the LAA ostium. Implant technique, pre-procedure evaluation, sizing, and troubleshooting have been reviewed, along with post implant follow-up and medication regimen detail. Future clinical trials (CATALYST trial, NCT\# 04226547) may support LAA occlusion for a wider population of AF patients who are not perceived to be at high bleeding risk.

\section{Figure and Video Legends}

Figure 1. Amplatzer Amulet LAAC device. Blue arrows denote lobe attachment and site of anchoring by stabilizing wires. Red arrows denote Amulet disc and position at PV ridge to LAA ostium. Panel A- 34mm device with angiogram showing occlusion of the LAA. Panel B- Schematic of target locationn. Panel C- TEE showing chronic seal with no leak or DRT at 1 year TEE.

Figure 2. Chart showing LAA occlusion options and corresponding LAA anatomy, clinical scenario, or patient medical history for which each option may be favored.

Figure 3. Sizing measurements based on disc ostium and LZ or landing zone for anchoring lobe. Yellow arrows denote the disc location tip of LPV-LAA ridge to LAA ostium edge at the mitral annulus. Red line indicates the location for measurement of anchoring lobe (LZ). A $25 \mathrm{~mm}$ Amulet was selected based on the LZ with max width of $22.6 \mathrm{~mm}$.

Figure 4. Example of over compression of anchoring lobe. Left panel A shows coaxial lobe position with obvious compression to the 'barrel' shape with minimal eversion of the distal pin. Red lines show lobe width. Panel B,C- Blue arrows denote lobe length. When the lobe length significantly exceeds width (2:1), repositioning is suggested or switch to smaller Amulet device.

Figure 5. Examples of disc location at risk for potential formation of device related thrombus (DRT). When angle is $>90$ degrees, risk is mitigated. Yellow arrow shows good seal of disc at mitral side. Red lines denote the angle of disc to PV ridge, also note measurement angle 36 degree on $\mathrm{L}$ panel, 53 degree on $\mathrm{R}$ panel. Blue arrow highlights potential DRT location.

Figure 6. Amulet device in the Torque Vue sheath and in 'ball' configuration noted by yellow arrow. Red line denotes target LZ region below which the lobe should be positioned. Solid black lines identify LAA neck. LCX $=$ Left circumflex artery.

Figure 7. Amulet lobe positions which should be repositioned. Panel A- TEE view with proximal location of lobe and separation from target tissues along mitral edge. Panel B- TEE with 3mm Doppler US color flow past lobe, correlates to Panel $\mathrm{C}$ and D showing off-axis lobe and low compression with angiogram confirming flow past the lobe.

Video 1. Three panels show sequential assembly from unopened package to de-airing the Amulet while submerged under saline bath to ensure risk for air embolism is minimized.

Video 2. Biplane TEE showing sheath position just inside LAA ostium and relatively midline to the LAA neck for lobe delivery.

Video 3a. Amulet going from ball position to triangle and then full lobe deployment with gentle cable advancement while keeping slight forward pressure on Torque Vue sheath.

Video 3b. Recapture of lobe back to the ball position to re-orient deployment.

Video 4a. Angiography through sheath with lobe deployed. 
Video 4b. Lobe position same location by TEE.

Video 5. Deployment of the LA Amulet disc with slight shift in orientation of the Amulet lobe.

Video 6. Tension testing of disc watching lobe position 30-60sec.

Video 7. Pulling disc back onto PV ridge, initial site too deep with risk for DRT.

Video 8. Release of Amulet device with snap back of disc, elliptical contour or concave appearance, separation of lobe and disc, disc and lobe off axis from each other, lobe coaxial to LAA neck and positioned below the LCX.

Video 9. TEE view of ideal position of LA disc on PV ridge, lobe compression, axis and location relative to LCX.

Video 10. Acute mild PV stenosis from Amulet disc pushing LPV-LAA ridge towards LSPV ostium.

\section{References}

1) January CT, Wann LS, Calkins H, et al. 2019 AHA/ACC/HRS Focused Update of the 2014 AHA/ACC/HRS Guideline for the Management of Patients With Atrial Fibrillation: A Report of the American College of Cardiology/American Heart Association Task Force on Clinical Practice Guidelines and the Heart Rhythm Society in Collaboration With the Society of Thoracic Surgeons. Circulation . 2019/07/09 2019;140(2):e125-e151. doi:10.1161/CIR.0000000000000665

2) January Craig T, Wann LS, Alpert Joseph S, et al. 2014 AHA/ACC/HRS Guideline for the Management of Patients With Atrial Fibrillation: Executive Summary. Journal of the American College of Cardiology . 2014/12/02 2014;64(21):2246-2280. doi:10.1016/j.jacc.2014.03.021

3) Holmes DR, Jr., Kar S, Price MJ, et al. Prospective randomized evaluation of the Watchman Left Atrial Appendage Closure device in patients with atrial fibrillation versus long-term warfarin therapy: the PREVAIL trial. J Am Coll Cardiol . Jul 8 2014;64(1):1-12. doi:10.1016/j.jacc.2014.04.029

4) Reddy VY, Doshi SK, Sievert H, et al. Percutaneous left atrial appendage closure for stroke prophylaxis in patients with atrial fibrillation: 2.3-Year Follow-up of the PROTECT AF (Watchman Left Atrial Appendage System for Embolic Protection in Patients with Atrial Fibrillation) Trial. Circulation . Feb 12 2013;127(6):720-9. doi:10.1161/circulationaha.112.114389

5) Kar S, Doshi SK, Sadhu A, et al. Primary Outcome Evaluation of a Next-Generation Left Atrial Appendage Closure Device: Results From the PINNACLE FLX Trial. Circulation. May 4 2021;143(18):17541762. doi:10.1161/circulationaha.120.050117

6) Lakkireddy D, Thaler D, Ellis CR, et al. Amplatzer Amulet Left Atrial Appendage Occluder Versus Watchman Device for Stroke Prophylaxis (Amulet IDE): A Randomized, Controlled Trial. Circulation . Nov 9 2021;144(19):1543-1552. doi:10.1161/circulationaha.121.057063

7) Reddy VY, Doshi SK, Sievert H, et al. Percutaneous Left Atrial Appendage Closure for Stroke Prophylaxis in Patients With Atrial Fibrillation. Circulation. 2013/02/12 2013;127(6):720-729. doi:10.1161/CIRCULATIONAHA.112.114389

8) Afzal Muhammad R, Gabriels James K, Jackson Gregory G, et al. Temporal Changes and Clinical Implications of Delayed Peridevice Leak Following Left Atrial Appendage Closure. JACC: Clinical Electrophysiology . 0(0)doi:10.1016/j.jacep.2021.06.018

9) Galea R, De Marco F, Meneveau N, Aminian A, Anselme F, Gräni C, Huber AT, Teiger E, Iriart X, Babongo Bosombo F, Heg D, Franzone A, Vranckx P, Fischer U, Pedrazzini G, Bedogni F, Räber L, Valgimigli M. Amulet or Watchman Device for Percutaneous Left Atrial Appendage Closure: Primary Results of the SWISS-APERO Randomized Clinical Trial.Circulation . 2021 Nov 6. doi: 10.1161/CIRCULATIONAHA.121.057859. PMID: 34747186 
Figure 1

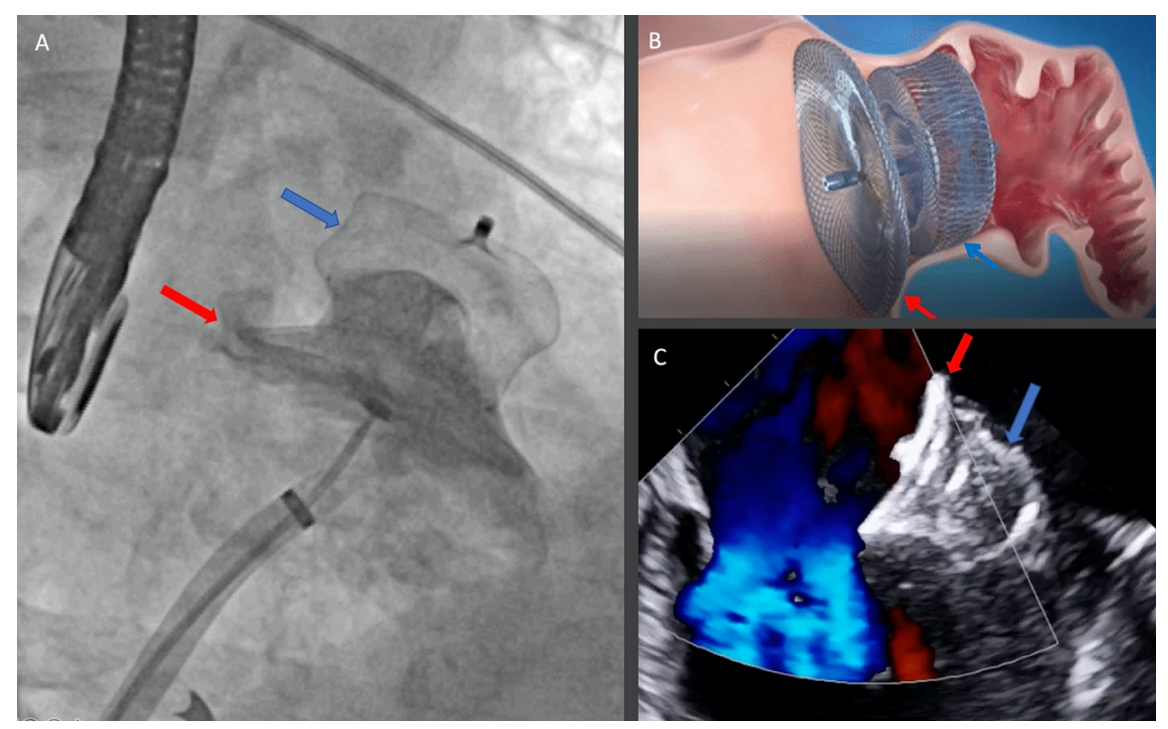

Figure 2

\section{Amulet LAA Occluder}

- Shallow implant depth $(10-15 \mathrm{~mm})$

- Large pits or lobes along the PV ridge

- LAA ostium $10-14 \mathrm{~mm}$ (with $16 \mathrm{~mm}$ device)

- LAA large and elliptical $\left({ }^{*} \mathrm{OI}>2\right)$

- Broccoli LAA with thick pectinates

- Obligation to DAPT

- Prior intra-cranial bleed

- Anchoring not stable with Watchman FLX

* $\mathrm{O}=$ = Ovality Index. Ratio of Largest LAA Dimeter at Ostium to Smalles Dimete.

\section{Lariat Sub-Xiphoid LAA Ligation}

- Windsock LAA and approach width $<45 \mathrm{~mm}$

- Proper thoracic anatomy for Sub-Xiphoid dry pericardial access

- Prior intra-cranial or near fatal bleeding

- Concomitant to hybrid AF ablation

- Known LAA triggers for AF

- Patient with extensive risk factor for DRT Avoid if prior adhesions, thoracic trauma, XRT, sternotomy or
pericarditis, chicken wing LAA behind the pulmonary artery

\section{Watchman FLX LAAC Device}

- Tolerates short term DOAC/OAC

- Most LAA anatomies with $>15 \mathrm{~mm}$ depth

- Patients with CKD stage III-IV

- Thrombocytopenia (can avoid DAPT)

- Frailty (risk for pericardial effusion, bleed)

- Patient can not do TEE (ICE guided implant)

- Prior CABG or incomplete S-LAAO

\section{Surgical LAAO (Suture, Atriclip)}

- Concomitant AVR/MVR, Coronary Bypass

- On or off pump surgical Cox-Maze III/IV

- Refractory DRT- LAAC device resection

- Recurrent LAA thrombus despite DOAC/OAC with clot evacuation

- Absolute contra-indications to OAC/DOAC

- Known LAA triggers for AF (Atriclip)

- LAA $>35 \mathrm{~mm}$ ostial width and LAAC indicated (too large for FLX, Amulet)

Figure 3 

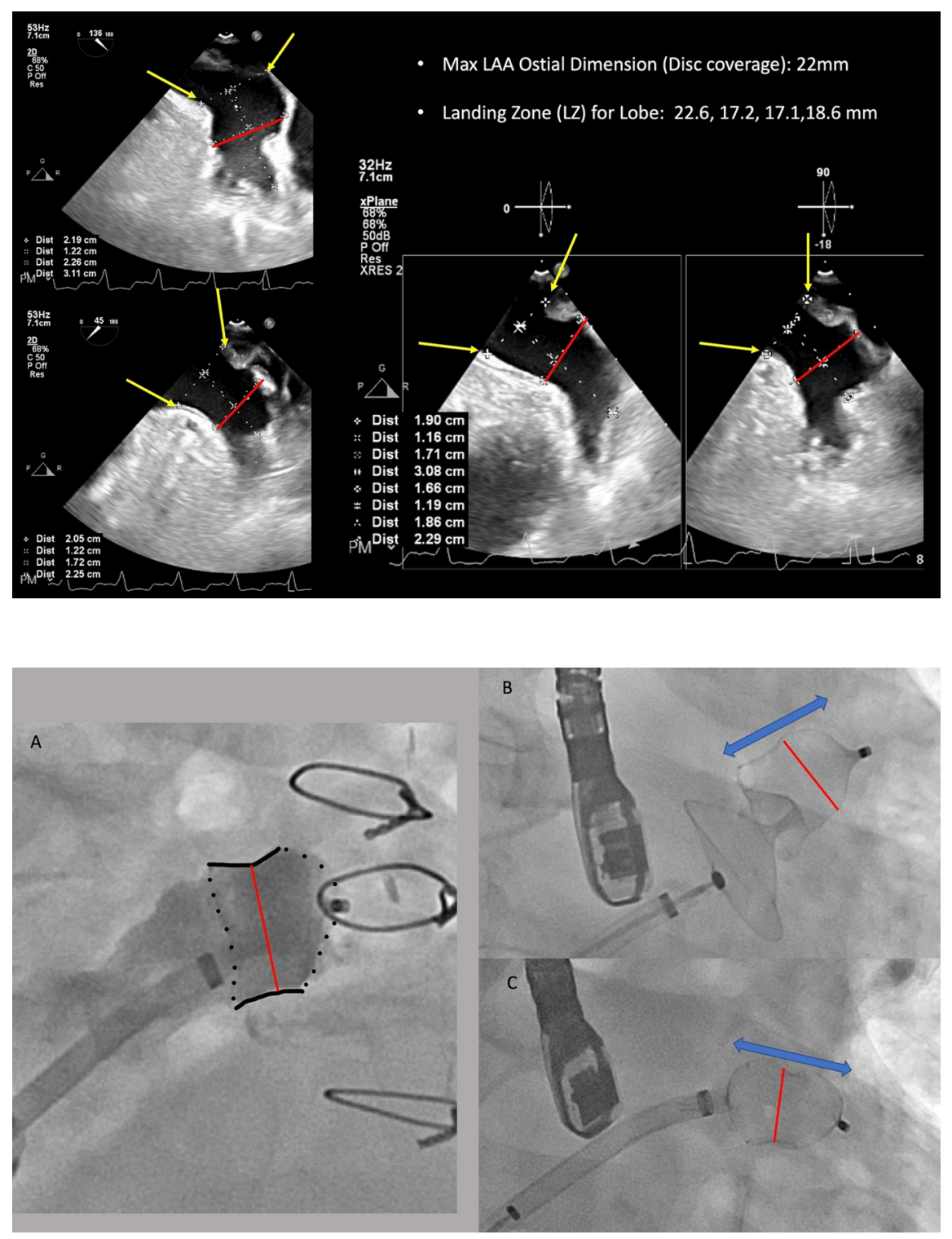

Figure 4

Figure 5 

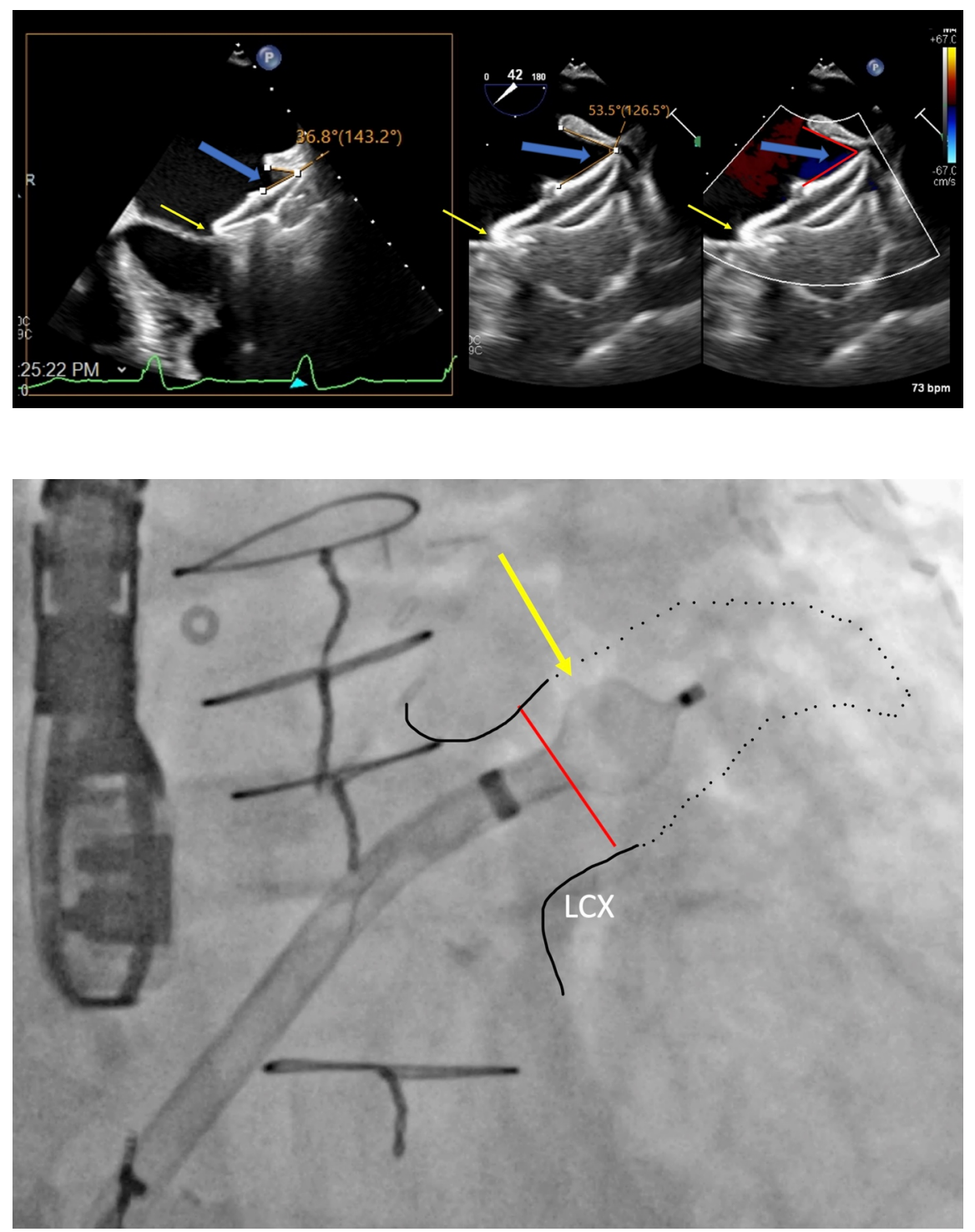

Figure 6

Figure 7 


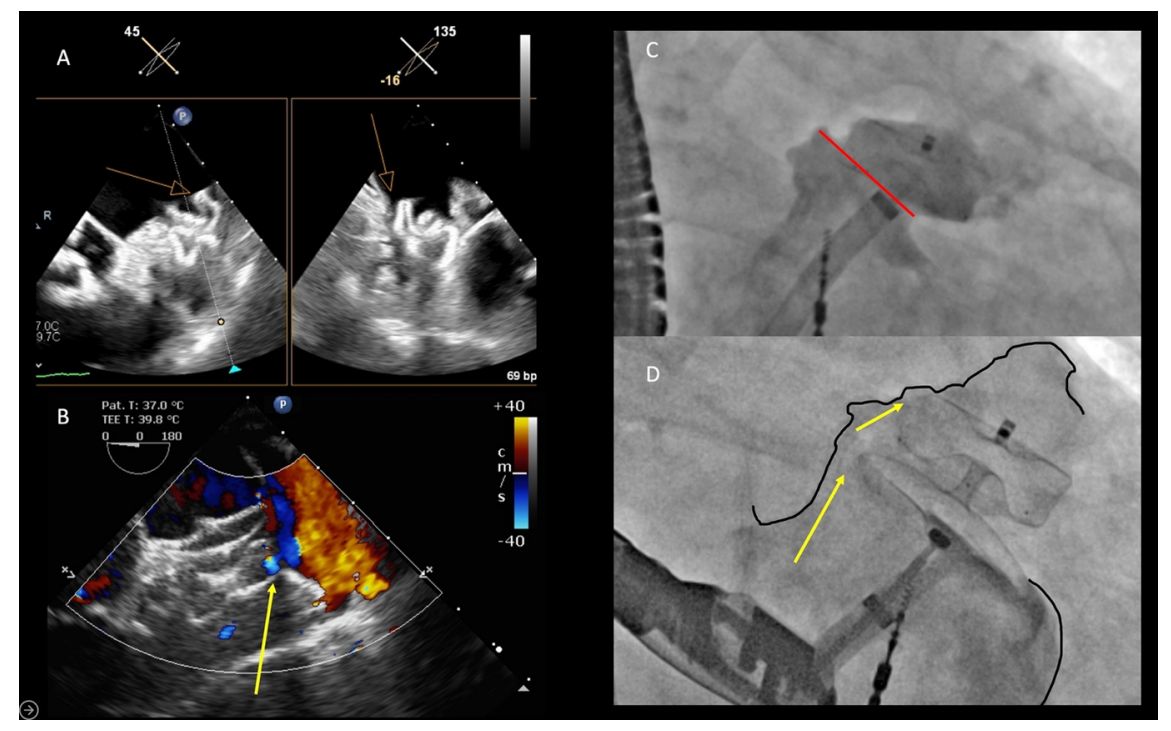

\section{Hosted file}

Video 1 Device Preparation.pptx available at https://authorea.com/users/457046/articles/ 553917-amplatzer-amulet-left-atrial-appendage-occluder-a-step-by-step-guide-to-deviceimplantation

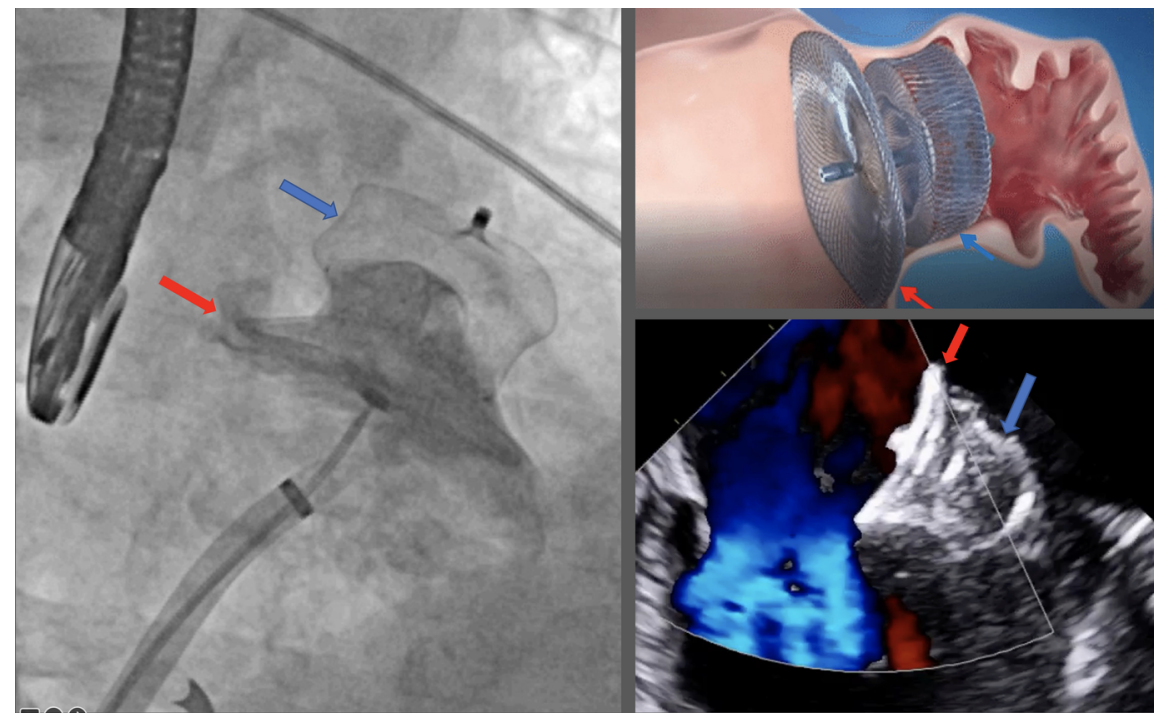




\section{Amulet LAA Occluder}

- Shallow implant depth $(10-15 \mathrm{~mm})$

- Large pits or lobes along the PV ridge

- LAA ostium $10-14 \mathrm{~mm}$ (with $16 \mathrm{~mm}$ device)

- LAA large and elliptical $\left({ }^{*} \mathrm{OI}>2\right)$

- Broccoli LAA with thick pectinates

- Obligation to DAPT

- Prior intra-cranial bleed

- Anchoring not stable with Watchman FLX

*OI = Ovality Index. Ratio of Largest LAA Dimeter at Ostium to Smallest Diameter.

\section{Lariat Sub-Xiphoid LAA Ligation}

- Windsock LAA and approach width $<45 \mathrm{~mm}$

- Proper thoracic anatomy for Sub-Xiphoid dry pericardial access

- Prior intra-cranial or near fatal bleeding

- Concomitant to hybrid AF ablation

- Known LAA triggers for AF

- Patient with extensive risk factor for DRT

Avoid if prior adhesions, thoracic trauma, XRT, sternotomy or pericarditis, chicken wing LAA behind the pulmonary artery
Watchman FIX LAAC Device

- Tolerates short term DOAC/OAC

- Most LAA anatomies with $>15 \mathrm{~mm}$ depth

- Patients with CKD stage III-IV

- Thrombocytopenia (can avoid DAPT)

- Frailty (risk for pericardial effusion, bleed)

Patient can not do TEE (ICE guided implant)

- Prior CABG or incomplete S-LAAO

\section{Surgical LAAO (Suture, Atriclip)}

- Concomitant AVR/MVR, Coronary Bypass

- On or off pump surgical Cox-Maze III/IV

- Refractory DRT- LAAC device resection

- Recurrent LAA thrombus despite DOAC/OAC with clot evacuation

- Absolute contra-indications to OAC/DOAC

- Known LAA triggers for AF (Atriclip)

- LAA >35mm ostial width and LAAC indicated (too large for FLX, Amulet)

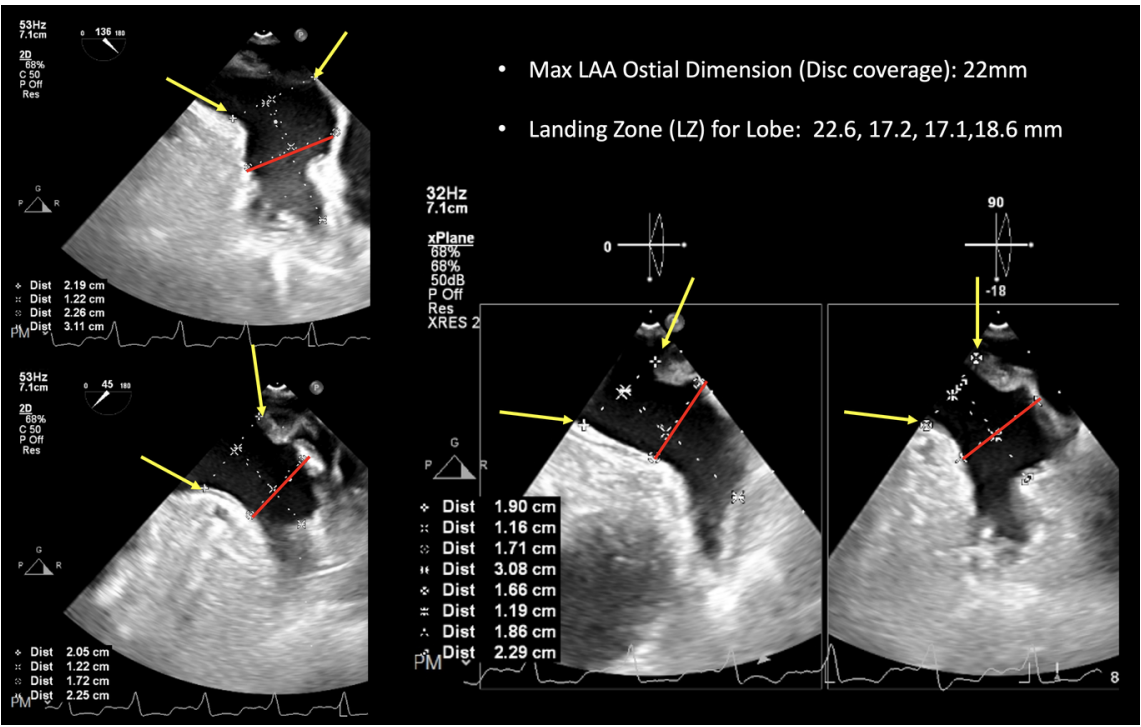




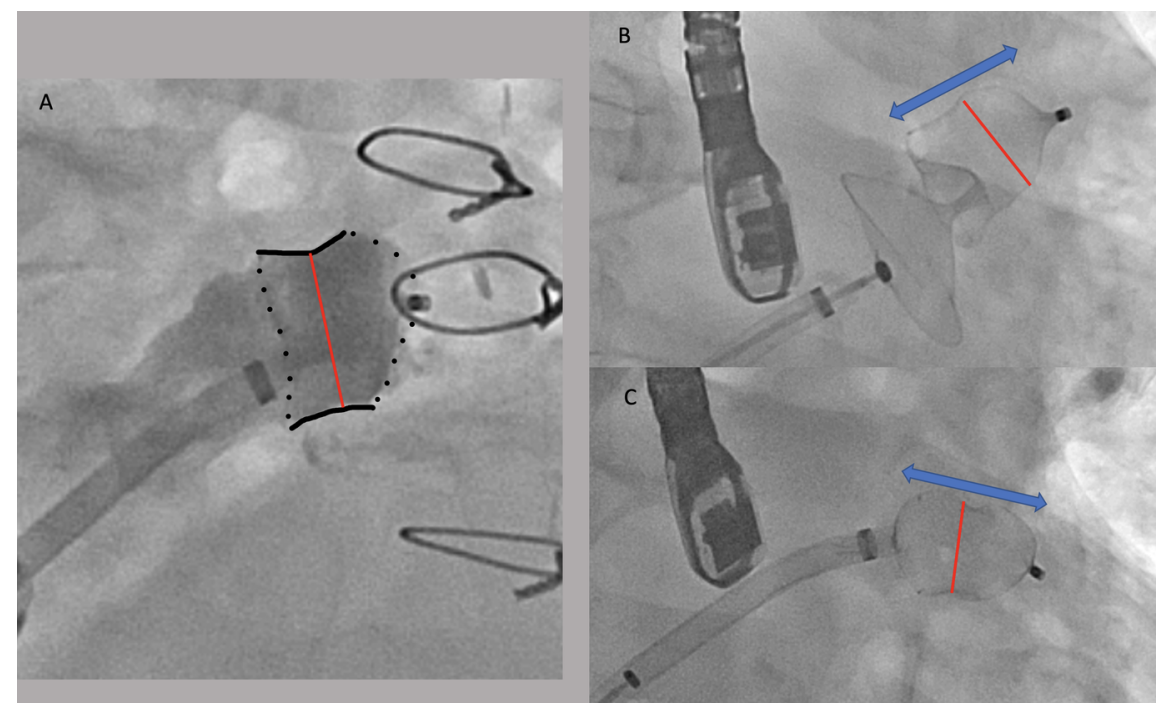

\section{Hosted file}

Video 7 Amulet pull disc to PV ridge.mp4 available at https://authorea.com/users/457046/ articles/553917-amplatzer-amulet-left-atrial-appendage-occluder-a-step-by-step-guide-todevice-implantation

\section{Hosted file}

Video 5 Amulet lobe shift with disc out.mp4 available at https://authorea.com/users/457046/ articles/553917-amplatzer-amulet-left-atrial-appendage-occluder-a-step-by-step-guide-todevice-implantation

\section{Hosted file}

Video 4a Amulet lobe angio before disc.mp4 available at https://authorea.com/users/457046/ articles/553917-amplatzer-amulet-left-atrial-appendage-occluder-a-step-by-step-guide-todevice-implantation

\section{Hosted file}

Video 6 tension test Amulet.mp4 available at https://authorea.com/users/457046/articles/ 553917-amplatzer-amulet-left-atrial-appendage-occluder-a-step-by-step-guide-to-deviceimplantation

\section{Hosted file}

Video 8 Amulet detach final.mp4 available at https://authorea.com/users/457046/articles/ 553917-amplatzer-amulet-left-atrial-appendage-occluder-a-step-by-step-guide-to-deviceimplantation

\section{Hosted file}

Video 3b recapture to ball.mp4 available at https://authorea.com/users/457046/articles/ 553917-amplatzer-amulet-left-atrial-appendage-occluder-a-step-by-step-guide-to-deviceimplantation

\section{Hosted file}


Video 10 Amulet disc with some PV stenosis $1 . \mathrm{mp} 4$ available at https://authorea.com/users/ 457046/articles/553917-amplatzer-amulet-left-atrial-appendage-occluder-a-step-by-stepguide-to-device-implantation

\section{Hosted file}

Video 9 Proper Amulet disc TEE.mp4 available at https://authorea.com/users/457046/articles/ 553917-amplatzer-amulet-left-atrial-appendage-occluder-a-step-by-step-guide-to-deviceimplantation

\section{Hosted file}

Video 3a Amulet ball to lobe deployment.mp4 available at https://authorea.com/users/457046/ articles/553917-amplatzer-amulet-left-atrial-appendage-occluder-a-step-by-step-guide-todevice-implantation

\section{Hosted file}

Video 4b Amulet lobe position by TEE before disc.mp4 available at https://authorea.com/ users/457046/articles/553917-amplatzer-amulet-left-atrial-appendage-occluder-a-step-bystep-guide-to-device-implantation

\section{Hosted file}

Video 2 TEE sheath position.mp4 available at https://authorea.com/users/457046/articles/ 553917-amplatzer-amulet-left-atrial-appendage-occluder-a-step-by-step-guide-to-deviceimplantation

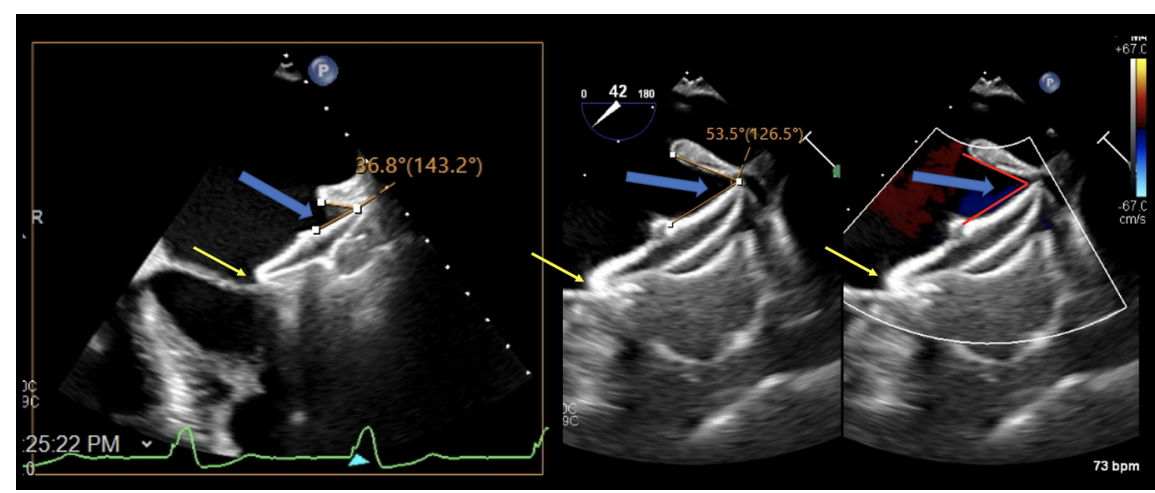



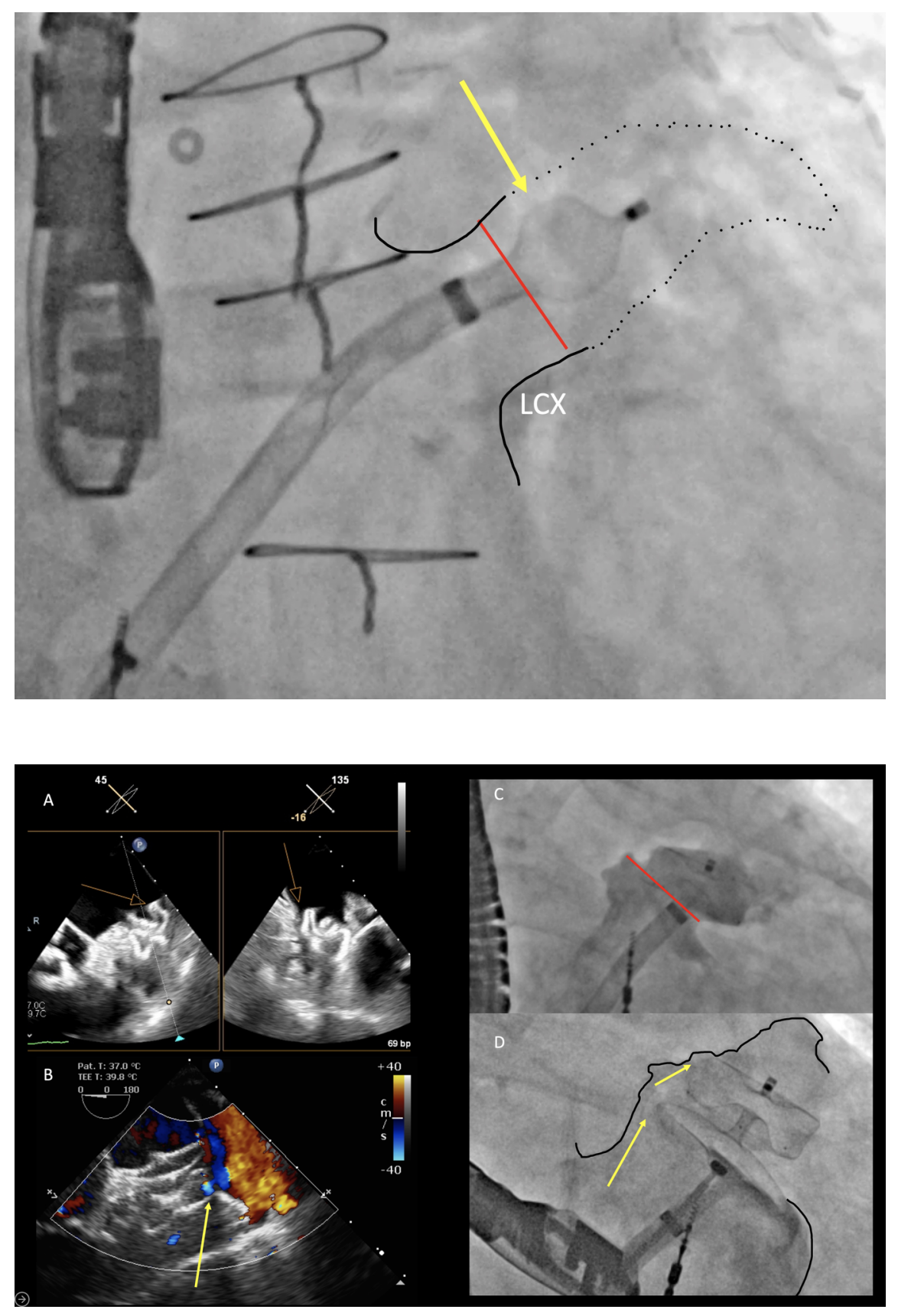